\title{
Extensive circumferential endoscopic mucosal resection with a new rigid esophagoscope: An animal study
}

\author{
Raphaelle Pilloud, MD, Yves Jaquet, MD, and Philippe Monnier, MD
}

\section{eonly Additional material is available online.}

From the Department of Otolaryngology, Head and Neck Surgery, Centre Hospitalier Universitaire Vaudois, Lausanne, Switzerland.

Supported by the Otolaryngology, Head and Neck Surgery Department Fund.

Preliminary results were presented at the annual meeting of the Swiss Society of Otolaryngology, Head and Neck Surgery, Lausanne, Switzerland, June 19-21, 2003.

Received for publication March 30, 2005; revisions received June 1, 2005; accepted for publication June 7, 2005.

Address for reprints: Raphaelle Pilloud, MD, Department of Otolaryngology, Head and Neck Surgery, Centre Hospitalier Universitaire Vaudois, CH-1011 Lausanne, Switzerland (E-mail: raphaelle.pilloud@hcuge.ch).

J Thorac Cardiovasc Surg 2005;130:1399-405 $0022-5223 / \$ 30.00$

Copyright $\odot 2005$ by The American Association for Thoracic Surgery

doi:10.1016/j.jtcvs.2005.06.038
Objective: Current endoscopic mucosal resection techniques are suboptimal for large circumferential mucosal resections intended to treat Barrett mucosa with multicentric foci of high-grade intraepithelial neoplasia or early adenocarcinomas. A rigid modified endoscope was developed for extended endoscopic mucosal resection in the esophagus. This pilot animal study investigated the feasibility of circumferential endoscopic mucosal resections of different lengths in the sheep esophagus.

Methods: Circumferential esophageal endoscopic mucosal resections of $2.2 \mathrm{~cm}(\mathrm{n}=$ 6), $3.3 \mathrm{~cm}(\mathrm{n}=6), 4.4 \mathrm{~cm}(\mathrm{n}=7)$, and $5.5 \mathrm{~cm}(\mathrm{n}=5)$ were performed in 24 sheep. Circumferential resections consisted of two opposite hemicircumferential mucosectomies. Animals were followed up with endoscopic examinations at 1 week and then monthly to 6 months, or until complete re-epithelialization without stenosis eventually ensued. Strictures were treated with a single or repeated dilatations with Savary bougies.

Results: Circumferential resections 2.2 to $5.5 \mathrm{~cm}$ in length were successfully performed in 23 of 24 of the animals. One perforation occurred directly after mucosectomy. Accurate depth of resection through the submucosa was obtained in $85 \%$ of the specimens. Cicatricial stenosis was controlled in $95 \%$ by bougienage.

Conclusion: Endoscopic mucosal resection performed with the rigid esophagoscope allowed extensive circumferential resections in a single session. There is thus potential to eradicate complete Barrett esophagus with high-grade intraepithelial neoplasia or early adenocarcinomas.

$\mathrm{T}$ The current treatment of choice with curative intent for high-grade intraepithelial neoplasia (HGIN) and early stage esophageal adenocarcinoma arising in Barrett esophagus (BE) is esophagectomy. ${ }^{1}$ Because of intensified endoscopic surveillance, the number of early-stage adenocarcinomas detected in the distal esophagus has recently increased. The prevalence of regional lymph node metastases of intramucosal adenocarcinomas (Tis, T1a) stands around 7\%, whereas it reaches as high as $50 \%$ when tumors invade the submucosa (T1b). ${ }^{2}$ This finding has raised questions regarding the extent of the surgery required to cure intramucosal adenocarcinoma and has prompted the use of nonsurgical therapeutic techniques to ablate dysplastic changes in BE and intramucosal adenocarcinoma, especially in elderly patients who are poor candidates for surgery.

Different techniques of endoscopic mucosal resection (EMR) with flexible endoscopes have been described and applied for localized carcinomas of the esophagus with good results. ${ }^{3-5}$ They are all associated with low morbidity and mortality. EMR has the advantage of providing a specimen for histologic examination. However, the resected surface of a single specimen does not exceed $2 \mathrm{~cm}^{2}$ with the commonly used cap-fitted flexible esophagoscope. For long-segment or circumferential lesions, piecemeal resections are therefore required. Long procedures of 


\section{Abbreviations and Acronyms \\ $\mathrm{BE}=$ Barrett esophagus \\ $\mathrm{EMR}=$ endoscopic mucosal resection \\ HGIN $=$ high-grade intraepithelial neoplasia}

several hours or multiple sessions are often necessary. Furthermore, a precise histologic mapping is less achievable, and the examination of the surgical margins is often unreliable. ${ }^{6-8}$ To overcome these drawbacks, many new techniques of large en bloc mucosal resection are currently in experimental or clinical trials, but no ideal method has yet been discovered. ${ }^{9-11}$ Either long procedures are still required or severe complications such perforations may occur. Cicatricial strictures are well-known consequences of circumferential resections in the esophagus but are amenable to treatment by repeated sessions of bougienage or balloon dilatation. $^{6,7}$

Since 1996 in our clinic, a new technique for EMR has been developed in collaboration with Karl Storz GmbH \& Co (Tüttlingen, Germany) ${ }^{12}$ to overcome the previously mentioned technical problems. It consists of a modified rigid esophagoscope that allows resection of a specimen varying in size from $4.4 \mathrm{~cm}^{2}$ to $12 \mathrm{~cm}^{2}$ as a single piece. A previous animal study of 55 hemicircumferential $4.4-\mathrm{cm}$ long resections showed a precise, deep resection margin through the submucosa, adequate histologic control of the resected specimen, and the absence of perforation or cicatricial stenosis. ${ }^{13}$ This study investigated the technical feasibility and safety of circumferential mucosal resections of different lengths with the EMR rigid esophagoscope in sheep.

\section{Materials and Methods \\ EMR Technique}

The EMR esophagoscope and technique of resection have been previously described in detail by Radu and colleagues. ${ }^{13}$ EMR with the rigid scope relies on the following principles: aspiration of the mucosa against a flat surface, precise cut at a regular depth through the submucosa of a large mucosal surface ranging from 2 to $11 \mathrm{~cm}^{2}$ with an electrocautery device, and visual control of the whole procedure (Figure E1).

The modified esophagoscope consists of a metallic outer tube $15 \mathrm{~mm}$ in diameter. At its distal end, a hemicircumferential aperture $6 \mathrm{~cm}$ in length has been designed. For introduction into the esophagus, an inner tube is placed inside the outer tube to seal its distal aperture and thus avoid potential mucosal tears. This step is carried out under visual control with a $0^{\circ}$ telescope located in the inner tube. Once at the level of the lesion, the inner tube is removed and replaced with the resectoscope. The extremity of the resectoscope is made of a transparent and perforated window opened on $180^{\circ}$ and adjusted to the aperture of the outer tube. A $30^{\circ}$ telescope located inside the resectoscope allows the accurate targeting of the lesion. Negative pressure is applied inside the

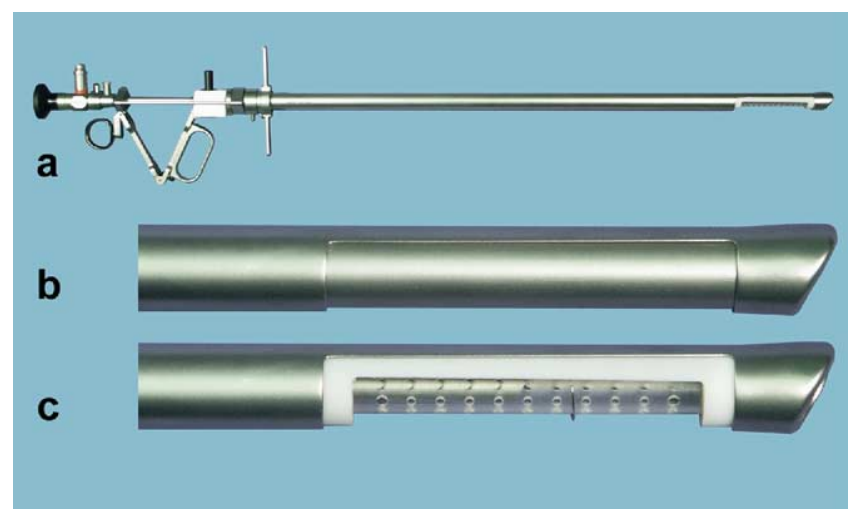

Figure 1. New rigid esophagoscope for mucosectomy. a, Large view. b, Outer tube is introduced in esophagus. Inner tube seals distal aperture. c, Cartridge and its transparent window $(5.5 \mathrm{~cm}$ in length) are placed inside outer tube. Resection wire loop moves from distal edge of window toward proximal edge.

resectoscope to aspirate the mucosa against the perforated window (Figure 1). An electric wire loop can be moved from the distal towards the proximal part of the window to cut the mucosa at a constant depth. The wire loop is connected to a high-frequency power device (Erbe-Tom T400; Erbe elektromedizin GmbH, Tübingen, Germany) initiated by foot control. The section of the electric wire loop is of small dimension $(0.3 \mathrm{~mm})$ and produces only minimal thermal damage. The constant gap of $1.0 \pm 0.1 \mathrm{~mm}$ between wire loop and transparent window determines the depth of resection. The endoscopist must pay special attention to carrying out the resection at a constant speed (wire loop shifting at about 1 $\mathrm{cm} / \mathrm{s}$ ). The resection is achieved in 5 to 10 seconds. After the resection, the piece of mucosa remains stuck against the transparent window and thus can be removed together with the endoscope. The single resected specimen is then stretched to its original size, pinned on a support, and fixed in $4 \%$ formalin for histologic examination.

Four different lengths of windows are available, allowing mucosal resections $2.2,3.3,4.4$, and $5.5 \mathrm{~cm}$ in length. The width of the resected area, defined by the hemicircumferential aperture of the window, is $2 \pm 0.2 \mathrm{~cm}$. A piece of mucosa of as large as 11 $\mathrm{cm}^{2}$ can be obtained in a single resection.

The depth of resection used was chosen for a precise cut throughout the submucosa. The lamina muscularis mucosae must be completely removed without damaging the muscularis propria.

\section{Animal Study}

Twenty-four sheep weighing 41 to $84 \mathrm{~kg}$ were used in this experimental study. The sheep esophagus is very similar to that of human beings with respect to the histologic organization of its wall. ${ }^{14,15}$ Moreover, the thicknesses of the separate layers are comparable between the species. The animals were divided into four groups according to the length of circumferential resection: $2.2 \mathrm{~cm}$ (group 1, $\mathrm{n}=6$ ), $3.3 \mathrm{~cm}$ (group 2, $\mathrm{n}=6$ ), $4.4 \mathrm{~cm}$ (group $3, \mathrm{n}=7$ ), and $5.5 \mathrm{~cm}$ (group $4, \mathrm{n}=5$ ). Circumferential mucosal resection consisted of two opposite hemicircumferential resections 
TABLE 1. Endoscopic results

\begin{tabular}{lcccccc}
\hline Group & No. & $\begin{array}{c}\text { Length of } \\
\text { resection }(\mathbf{c m})\end{array}$ & $\begin{array}{c}\text { Mean dilatations } \\
\text { at } \mathbf{3} \text { mo }\end{array}$ & $\begin{array}{c}\text { Mean dilatations at } \\
\text { final follow-up }\end{array}$ & $\begin{array}{c}\text { Mucosal healing without } \\
\text { stenosis (mo.) }\end{array}$ & $\begin{array}{c}\text { Stenosis at end of } \\
\text { follow-up (No.)* }\end{array}$ \\
\hline 1 & 6 & 2.2 & $1.3(0-3)$ & $1.3(0-3)$ & $3.0 \dagger$ & $0 / 6$ \\
2 & 6 & 3.3 & $1.0(0-3)$ & $2.0(0-5)$ & $4.0(3-5)$ & $0 / 5$ \\
3 & 7 & 4.4 & $1.0(0-2)$ & $1.4(0-2)$ & $3.4(3-4)$ & $0 / 5$ \\
4 & 5 & 5.5 & $0.8(0-2)$ & $2.6(0-5)$ & $4.8(3-7)$ & $1 / 5$ \\
\hline
\end{tabular}

Values are means with ranges given in parentheses. *All animals. †Of living animals.

without previous saline injection of the submucosa to test the reliability of the in-depth resection. Thin residual mucosal strips of squamous cell epithelium were often found between the two resected areas. These were easily removed under visual control with a standard biopsy forceps to obtain a complete $360^{\circ}$ resection.

After the EMR, all animals were included in the same follow-up protocol: 1 week after the circumferential EMR, the animals again underwent endoscopy to ascertain whether any delayed perforation occurred and to remove the impacted straw that was often found in the early phase (first week) after circumferential EMR. Further follow-up endoscopies were performed monthly after the mucosectomy. If cicatricial stricture formation occurred, dilatation was performed by inserting Savary bougies of increasing sizes to $17 \mathrm{~mm}$ in diameter. Removal of impacted straw over the raw surface of the resected area was often needed before bougienage because sheep are ruminants. The animals were followed up with routine endoscopies until 6 months after the resection or until complete re-epithelialization without stenosis ensued. The length of the mucosal defect was measured during every endoscopic session. Absence of stenosis was defined when the passage of a universal rigid Storz esophagoscope $(15 \mathrm{~mm}$ in diameter) was possible without any resistance or mucosal tearing.

All endoscopic procedures were performed with the animals under general anesthesia. After administration of xylazine $(0.4$ $\mathrm{mg} / \mathrm{kg}$ intramuscularly), anesthesia was induced with thiopental (10 $\mathrm{mg} / \mathrm{kg}$ intravenously). Anesthesia was maintained by inhalation of halothane and mechanical ventilation through an orotracheal tube. After the mucosectomy, the sheep received nonsteroidal antiinflammatory treatment (phenylbutazone, $1 \mathrm{~g} / \mathrm{d}$ intramuscularly) for 2 weeks. After the EMR, animals were fed with a special smooth diet consisting of fresh grass. This diet was maintained for a period of 3 weeks to minimize the risk of injury and impaction of straw at the treated area. A normal diet of hay and straw was resumed afterward. During the duration of the experiments, the animals were housed in environmentally controlled facilities. At the end of the follow-up period, they were painlessly killed with an overdose of sodium pentobarbital. All experiments were done in accordance with protocols approved by the Ethics Committee for Animal Experiments of our institution.

\section{Histologic Analysis}

Resected specimen. All mucosectomy specimens were fixed in $4 \%$ buffered formalin, embedded in paraffin, longitudinally sectioned, and stained with hematoxylin-eosin. Histologic slides were analyzed for the depth and regularity of mucosal resection throughout the longitudinal section. The presence of the lamina muscularis mucosae on every slide attested to the submucosal resection. Islets of muscularis propria were also recorded when present.

Esophagus after complete re-epithelialization. After the animal had been killed, the treated segment of esophagus was measured for original length in situ and then harvested. The resected segment was then stretched to its original length and fixed in $4 \%$ formalin. Serial longitudinal sections, taken from the whole circumference, included the healed area and the adjacent untreated esophageal wall. The multiple sections were embedded in paraffin and stained with hematoxylin-eosin. The length of the re-epithelialized area, characterized by the lack of lamina muscularis mucosae, was measured. The depth and extent of scarring through the submucosa and muscularis propria were evaluated by an experienced senior pathologist. The longitudinal length of fibrosis encompassing more than $50 \%$ in thickness of the muscularis propria was measured and compared with the length of the re-epithelialized segment.

\section{Results}

Circumferential EMRs were done in 24 sheep by performing two opposite hemicircumferential resections. Thin residual mucosal strips persisted after the two opposite resections in 19 of 24 animals (79\%) but were easily removed with standard biopsy forceps. Total surgical time ranged between 20 and 30 minutes. This variation resulted chiefly from the frequent need to remove residual mucosal strips. Minor to moderate bleeding (to $150 \mathrm{~mL}$ ) was observed after the resection and was successfully controlled with icy water only. No handling problems were encountered during the procedure. Anesthesia was well tolerated in all cases.

Immediate postoperative endoscopy attested to the circumferential mucosal removal over the full length of the treated segment (Figure E2). Proximal and distal resection margins were sharply cut and stretched widely apart. The rectangular resected specimens were of constant thickness macroscopically. One week after the mucosectomy, the treated area was covered by various amounts of fibrin. Food impactions were found at this stage in 8 of 23 animals. Two impactions each occurred in groups 2 and 3. Four impactions were seen in group 4. All were easily removed with a standard biopsy forceps during endoscopy. One month after the operation, weight loss of $2 \%$ to $10 \%$ of the body mass was recorded, with complete recovery at 3 months. No food 


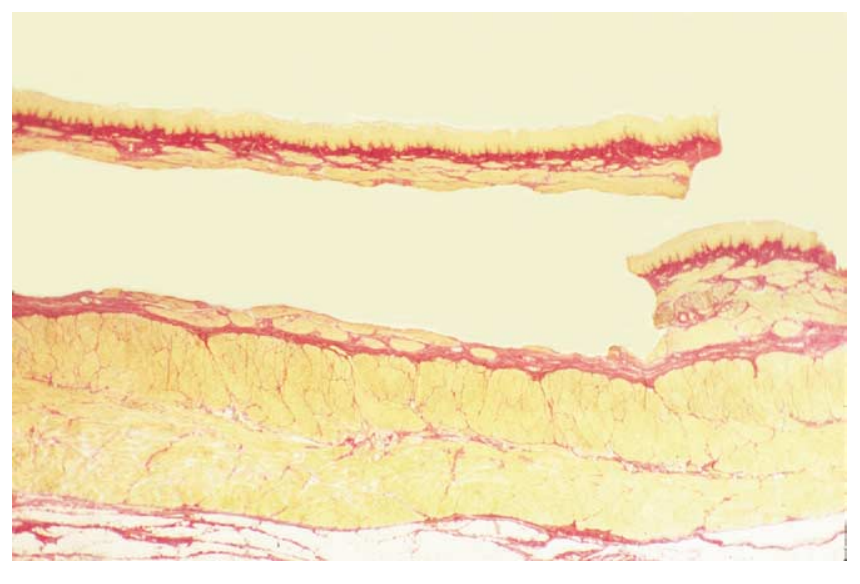

Figure 2. Resected specimen and corresponding esophageal segment (Van Gieson staining). Transverse section with resected specimen facing its corresponding esophageal wall. Cut was performed at regular depth through submucosa.

impaction was encountered after full re-epithelialization of the esophagus.

\section{Group 1: Circumferential Resection of $2.2 \mathrm{~cm}(\mathrm{n}=6)$} All 6 animals survived the follow-up period of 6 months until they were killed as planned. The mean tissue defect measured immediately after mucosectomy was $5.7 \mathrm{~cm}$ in length (range 5-7 cm). This corresponds to an increase of 2.6-fold compared with the length of the primary resection. Complete re-epithelialization was achieved in all cases after 3 months (Table 1). One animal had an early stenosis at 1 week that required dilatation. Three animals were dilated at 1 month, and 4 animals at 2 months. Two animals did not require any endoscopic dilatation. The mean number of dilatations needed per animal was 1.3 (Table 1). At 6 months of follow-up, no residual stenosis was encountered in the whole group.

\section{Group 2: Circumferential Resection of $3.3 \mathrm{~cm}(\mathrm{n}=6)$}

The mean postoperative length of tissue defect was $6.8 \mathrm{~cm}$, about twice the length of primary resection. One animal died during the follow-up period, at 2 months. Perforation of the esophagus was found at autopsy, but the cause remains unclear. Impaction of straw constitutes the most probable hypothesis. Complete re-epithelialization was observed in all other animals at 4 months. Three animals did not need any dilatation during the follow-up and healed without stenosis. One stenosis was seen at 1 week and consequently treated. Successive dilatations at 1, 2, 3, and 4 months were then necessary, and this animal was totally cured at 5 months. One animal needed four consecutive dilatations and ended up with absence of stenosis at 5 months. The mean number of dilatations needed per animal was 2 (range 0-5). At the end of the follow-up, no residual stenosis was encountered. The mean follow-up period necessary to achieve a normal esophageal lumen without stenosis was 4 months.

\section{Group 3: Circumferential Resection of $4.4 \mathrm{~cm}(\mathrm{n}=7)$}

Two animals died of perforations. The first death occurred the day after the mucosectomy and was directly related to the surgery. An overlap of the two opposing resections seems the most probable cause of this perforation. The second perforation was caused at 1 month by overly aggressive dilatation (bougienage from $9-18 \mathrm{~mm}$ ) for a tight stenosis. The remaining 5 animals had a mucosal defect averaging $7 \mathrm{~cm}$ in length (range 6-9 $\mathrm{cm}$ ) after EMR, corresponding to a 1.6-fold expansion. Re-epithelialization was complete at 4 months in all animals. One animal healed completely without the need for any dilatation. One animal required only a single dilatation at 2 months and was cured of its stenosis at 3 months. Two dilatations per animal were necessary to treat the 3 remaining animals. One needed two successive dilatations at 1 and 2 months and was cured at 3 months. The other two animals were dilated at 1 and 3 months and ended up with absence of stenosis at 4 months. The mean number of dilatations per animal was 1.4 (range: 0-2). At the end of the follow-up, absence of residual stenosis was found in all cases. The mean follow-up period necessary to achieve a normal esophageal lumen without stenosis was 3.4 months.

\section{Group 4: Circumferential Resection of $5.5 \mathrm{~cm}(\mathrm{n}=5)$}

All animals survived the follow-up period until they were killed as planned. No perforations were encountered. After mucosectomy, the mean length of tissue defect was $9 \mathrm{~cm}$ (range 8-9 cm), corresponding to a 1.6-fold increase. Complete re-epithelialization was achieved at 4 months in all animals. Only a single residual stenosis was present at 6 months of follow-up. The mean number of dilatations per animal was 2.6 (range 0-5). Two animals did not need any dilatation and were totally cured after 3 months. Three dilatations at 1,2 , and 3 months were performed in 1 animal, with a complete healing of the stenosis at 4 months (Figure E2). Two animals had to be treated with five successive dilatations at 1, 3, 4, 5, and 6 months. After 7 months, 1 of these 2 animals did not show any residual stenosis. The second had a mild stenosis $11 \mathrm{~mm}$ in diameter. Although this stenosis was considered incurable, the animal looked perfectly healthy and maintained a constant, normal weight.

\section{Histologic Analysis of Resected Specimens}

All 48 resected specimens of the 24 sheep that were followed up until the end of the experiment were analyzed microscopically (Figure 2). The layer of muscularis muco- 
sae was perfectly outlined in all specimens. An accurate depth of resection was obtained in 41 of 48 cases (85\%). The cut was regular and performed straight through the submucosa, without intrusion into the muscularis propria. In the remaining 8 specimens (15\%), scarce and superficial fibers of the internal layer of the muscularis propria were seen focally. It must be emphasized that the resections were done without previous saline injection of the submucosa.

\section{Histologic Analysis of Healed Esophagus}

The esophagi of the surviving sheep $(\mathrm{n}=21)$ were studied for long-term tissue damage and scarring processes. In the untreated sites, all anatomic layers of the esophageal wall were easily recognized. The thickness of the esophageal wall increased significantly over the treated segment (Figure E3). A morphologically normal epithelium was found at the re-epithelialized area in each specimen. Scarring of the lamina propria, absence of muscularis mucosae, and submucosal fibrosis characterized this re-epithelialized segment. The length of the area without lamina muscularis mucosae correlated with the initial length of resection (group 1) or was slightly shorter (groups 2 to 4; Table E1).

Fibrosis of the muscularis propria exceeded $50 \%$ of its thickness over about $25 \%$ of the resection length in each group (Table E1). The peak of fibrosis was situated at the midpoint between the upper and lower resection margins (documented by the interruption of the lamina muscularis mucosae) and often encompassed the full thickness over a very limited segment $(1-3 \mathrm{~mm})$.

\section{Discussion}

The widespread use of gastroesophageal endoscopy in Western countries has led to the detection of a fair number of dysplastic changes and early-stage adenocarcinomas in the distal esophagus. Currently, the safest treatment modality for HGIN in BE is esophagectomy. ${ }^{1}$ Unfortunately, this surgical procedure is linked with a significant mortality and morbidity, especially for elderly patients who are often poor candidates for surgery. ${ }^{16,17}$ Eradication of dysplastic BE by endoscopic means is thus appealing, but it must meet all of the following basic criteria: (1) The segment of intestinal metaplasia must be resected completely over its entire length, not only on the mucosal surface but also in depth. (2) Histologic analysis of the resected specimen must be possible. (3) A precise orientation of the specimen is mandatory for a reliable evaluation of the surgical margins. For this purpose, the number of resected specimens should be no more than one or two. (4) The re-epithelialization should take place with squamous cell mucosa. A nonacidic environment is therefore mandatory. (5) The formation of esophageal stenosis must be avoided.

These five criteria are still not fulfilled with the EMRflexible gastroscope in cases of circumferential short- and long-segment BE. Satodate and associates ${ }^{7}$ have reported the successful treatment of circumferential $\mathrm{BE}$ by the piecemeal removal of more than 30 resected specimens during a very long procedure. Giovannini and colleagues ${ }^{8}$ also described the use of piecemeal circumferential EMR in 21 patients with early lesions of BE. The mean number of pieces removed was 4.5 per session, and two sessions were needed to perform the circumferential resection. Needless to say, such endoscopic performances are tedious in daily practice. Furthermore, a precise histologic study to ensure a complete resection with negative margins was almost impossible in these cases, and the need for several EMRs during the same session may have increased the rate of perforation by overlaps of resections, although this has not been reported.

Although techniques of widespread EMR with flexible endoscopes are emerging in clinical practice and allow somewhat larger EMR areas, multiple resections are often still required to cure extended lesions, and the procedures remain long. ${ }^{9}$ Furthermore, a significantly high level of technical skill seem to be mandatory, ${ }^{10}$ and severe complications tend to appear. ${ }^{11}$

\section{Rigid Esophagoscope for Mucosectomy}

The patented rigid esophagoscope for mucosectomy was developed in collaboration with Karl Storz GmbH \& Co. It fulfills all basic requirements for a safe resection of the full thickness of the Barrett mucosa, including the lamina muscularis mucosae. According to the size of the distal window, resected specimens ranging from $2 \mathrm{~cm}^{2}$ to $12 \mathrm{~cm}^{2}$ can be obtained in a single piece and at a constant depth of $1.0 \pm$ $0.1 \mathrm{~mm}$. Each resection takes no longer than 10 seconds. Technically, a circumferential resection by two opposite EMRs is easily feasible and thus allows the resection of a 6-cm long circumferential BE in a single endoscopic session of less than 30 minutes. To our knowledge, no other endoscope currently achieves such a degree of performance. In this animal study, long-segment circumferential resections (as great as $11 \mathrm{~cm}^{2}$ ) were performed without technical difficulties.

\section{Macroscopic and Histologic Analysis of Resected Specimens and Healed Esophageal Specimens}

After EMR, immediate measurement of the tissue defect showed a 1.6- to 2.6-fold increase in the length of the primary resection, according to the size of the window used for the resection. This can be explained by the retraction of the mucosa at both ends of the resected area.

The orientation of the rectangular specimen of mucosa was straightforward, with proximal and distal ends easily identified. This allowed an accurate histologic examination by the pathologist, ensuring correct analysis of the resection margins. 
In all specimens, the depth of resection was constant throughout the submucosa, even for the longest resections $(5.5 \mathrm{~cm})$. Histologically, the lamina muscularis mucosae were present throughout all specimens. Considering that the mean thickness of Barrett mucosa is about $0.5 \mathrm{~mm}$ (range $0.39-0.59 \mathrm{~mm}),{ }^{18}$ our instrument should be adequate to treat BE without leaving deep glands behind and without damaging the muscularis propria.

Healed esophagi. Only 1 residual stenosis (5\%), which was moderate ( $11 \mathrm{~mm}$ in diameter), was seen among the 21 surviving sheep. The histologic analysis revealed scarring of the muscularis propria reaching the outer layer over a segment of esophagus that corresponded to approximately $25 \%$ of the primary length of resection in all animals. This observation could not be explained by thermal damage induced by the diathermal wire loop moving at a constant speed from the distal to the proximal end of the resection window. Instead, the mechanism was most probably related to the long exposure (as long as 4 months) of the muscularis propria to the passage of the food bolus and refluxate through the esophagus of the sheep. Proton-pump inhibitors were not used in this study but might have been of benefit to reduce the inflammation process leading to stenosis. Although 13 of 21 animals had a stenosis at some point during the experiment, residual stenosis was prevented in 20 of 21 animals by means of 1 or more dilatation (maximum 5). Future trials should investigate other chemical or even physical methods to suppress the process of stricture formation. Nevertheless, it is probably beyond any hope that scarring of the muscularis propria of the esophageal wall can be avoided entirely when extensive circumferential resections are carried out.

\section{Complications}

The overall mortality was 13\% (3/24 animals). Two perforations occurred during the follow-up period. One perforation at 1 month was due to an overly aggressive dilatation. The stenosis was very tight, but nonetheless bougienage to $18 \mathrm{~mm}$ was performed. The second perforation occurred at 2 months without any precipitating event. An impaction of straw in the weakened resected area most probably led to the perforation. As such, the overall mortality is still higher than that commonly reported for resection of the esophagus, but unexpected perforation during the healing phase is less likely in human beings with a better adaptation of their diet. Special care must be taken in the follow-up period to reduce the rate of complications. Especially, dilatation of stenosis must be performed with an increment in the size of bougies that is adapted to the tightness of the stenosis and reaching a maximum $15 \mathrm{~mm}$. In human beings, this corresponds to the size for a normal dietary intake without dysphagia. Moreover, solid food should be avoided until complete re-epithelialization has occurred.
The rate of perforation assumed to be directly related to the EMR technique was $4.2 \%$ (1/24 animals). The single perforation occurred the day after the mucosectomy. It must be noted however, that the resections were carried out without previous submucosal injections, to assess the reliability of the instrument for submucosal resections, and that a lateral overlap of the windows of resection most probably explained this complication. With a systematic submucosal injection of saline solution and epinephrine, the risk of perforation should further decrease.

\section{Perspectives}

As it has been designed, the EMR rigid esophagoscope is a suitable instrument for studying and staging epithelial lesions in the esophagus. The distal window, with its disposable cartridge, allows the resection of mucosal specimens of different sizes $\left(2-12 \mathrm{~cm}^{2}\right)$. In human beings, this EMR rigid esophagoscope will allow precise staging of early esophageal cancers (squamous cell carcinoma or adenocarcinoma), more accurate ultimate diagnosis of dysplastic BE (in comparison with multiple biopsies performed at random), potentially curative treatment of squamous cell carcinoma without in-depth invasion beyond the lamina muscularis mucosae, and curative treatment of dysplastic intestinal metaplasia or early adenocarcinoma arising in $\mathrm{BE}$.

\section{Conclusion}

The approach of a minimally invasive treatment modality by the endoscopic resection of superficial esophageal cancers can only be envisaged if adequate resected specimens are available for a precise histologic analysis of the lateral and deep resection margins. The potential role of this EMR rigid esophagoscope is thus interesting, because currently it is the only instrument that quickly allows the resection of mucosal specimens (as large as $12 \mathrm{~cm}^{2}$ ) in a single piece and at a constant depth at the level of the submucosa. Clinical trials will be initiated shortly to assess the real potential of this instrument in human beings.

\section{References}

1. Sampliner RE, Practice Parameters Committee of the American College of Gastroenterology. Updated guidelines for the diagnosis, surveillance, and therapy of Barrett's esophagus. Am J Gastroenterol. 2002;1997:1888-95.

2. Nigro JJ, Hagen JA, DeMeester TR, DeMeester SR, Peters JH, Oberg $\mathrm{S}$, et al. Prevalence and location of nodal metastases in distal esophageal adenocarcinoma confined to the wall: implications for therapy. J Thorac Cardiovasc Surg. 1999;117:16-25.

3. Ell C, May A, Gossner L, Pech O, Gunter E, Mayer G, et al. Endoscopic mucosal resection of early cancer and high grade dysplasia in Barrett's esophagus. Gastroenterology. 2000;118:670-7.

4. May A, Liebwin G, Behrens A, Kohnen R, Viet M, Stolte M, et al. A prospective randomized trial of two different endoscopic resection techniques for early stage cancer of the esophagus. Gastrointest Endosc. 2003;58:167-75.

5. Inoue H, Takeshita K, Hori H, Muraoka Y, Yoneshima H, Endo M. Endoscopic mucosal resection with a cap-fitted panendoscope for 
esophagus, stomach and colon mucosal lesions. Gastrointest Endosc. 1993;39:58-62.

6. Seewald S, Akaraviputh T, Seitz U, Brand B, Groth S, Mendoza G. Circumferential EMR and complete removal of Barrett's epithelium: a new approach to management of Barrett's esophagus containing highgrade intraepithelial neoplasia and intramucosal carcinoma. Gastrointest Endosc. 2003;57: 854-9.

7. Satodate H, Inoue H, Yoshida T, Ususui S, Iwashita M, Fukami N, et al. Circumferential EMR of carcinoma arising in Barrett's esophagus: case report. Gastrointest Endosc. 2003;58:288-92.

8. Giovannini M, Bories E, Pesenti C, Moutardier V, Monges G, Danisi $\mathrm{C}$, et al. Circumferential endoscopic mucosal resection in Barret's esophagus with high-grade intraepithelial neoplasia or mucosal cancer: preliminary results in 21 patients. Endoscopy. 2004;36:782-7.

9. Rösch T, Sarbia M, Schumacher B, Deinert K, Frimberger E, Toermer $\mathrm{T}$, et al. Attempted endoscopic en bloc resection of mucosal and submucosal tumors using insulated-tip knives: a pilot series. Endoscopy. 2004;36:788-801.

10. Kuwano H, Mochiki E, Asao T, Kato H, Shimura T, Tsutsumi S. Double endoscopic Intraluminal operation for upper digestive tract diseases: proposal of a novel procedure. Ann Surg. 2004;239:22-7.
11. Rajan E, Gostout CJ, Feitoza AB, Leontovich ON, Herman LJ, Burgart LJ, et al. Widespread EMR : a new technique for removal of large areas of mucosa. Gastrointest Endosc. 2004;60:623-7.

12. Grosjean P. La mucosectomie endoscopique oesophagienne: étude pré-clinique. Akt Prob Otolaryngol. 1998;21:293-302.

13. Radu A, Grosjean P, Fontolliet C, Monnier P. Endoscopic mucosal resection in the esophagus using a new rigid device: an animal study. Endoscopy. 2004;36:298-305.

14. Liu JB, Miller LS, Goldberg BB, Feld RI, Alexander AA, Needleman L, et al. Transnasal US of the esophagus: preliminary morphologic and function studies. Radiology. 1992;184:721-7.

15. Taniguchi DK, Martin RW, Trowers EA, Silverstein FE. Simultaneous M-mode echoesophagram and manometry in the sheep esophagus. Gastrointest Endosc. 1995;41:582-6.

16. Kirby JD. Quality of life after oesophagectomy: the patients' perspective. Dis Esophagus. 1999;12:168-71.

17. Zaninotto G, Parenti AR, Ruol A, Costantini M, Merigliano S, Ancona E. Oesophageal resection for high-grade dysplasia in Barrett's esophagus. Br J Surg. 2000;87:1102-5.

18. Ackroyd R, Brown NJ, Stephenson TJ, Stoddard CJ, Reed MW. Ablation treatment for Barrett esophagus: what depth of tissue destruction is needed? J Clin Pathol. 1999;52:509-12. 


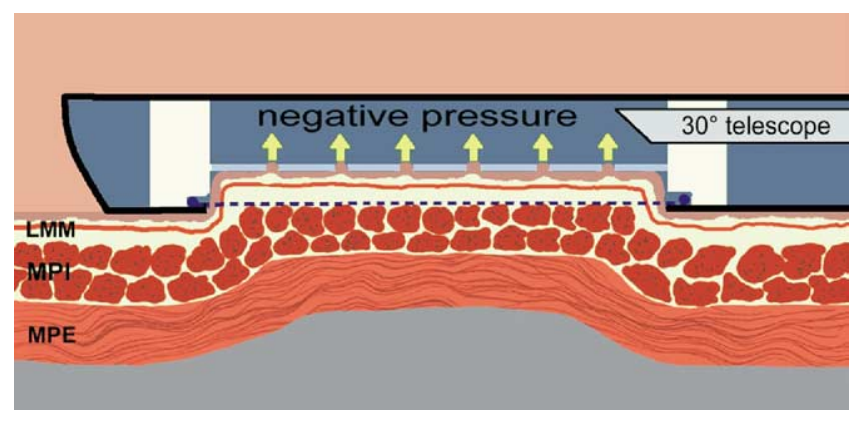

Figure E1. Principle. Negative pressure is applied inside resectoscope, and mucosa is sucked against perforated transparent window. Deep section (dashed lines) is performed at regular depth throughout submucosa. Lesion to be removed is located through $30^{\circ}$ angulated telescope, which also allows control of aspiration. $L M M$, Lamina muscularis mucosae; MPI, muscularis propria interna; MPE, muscularis propria externa.
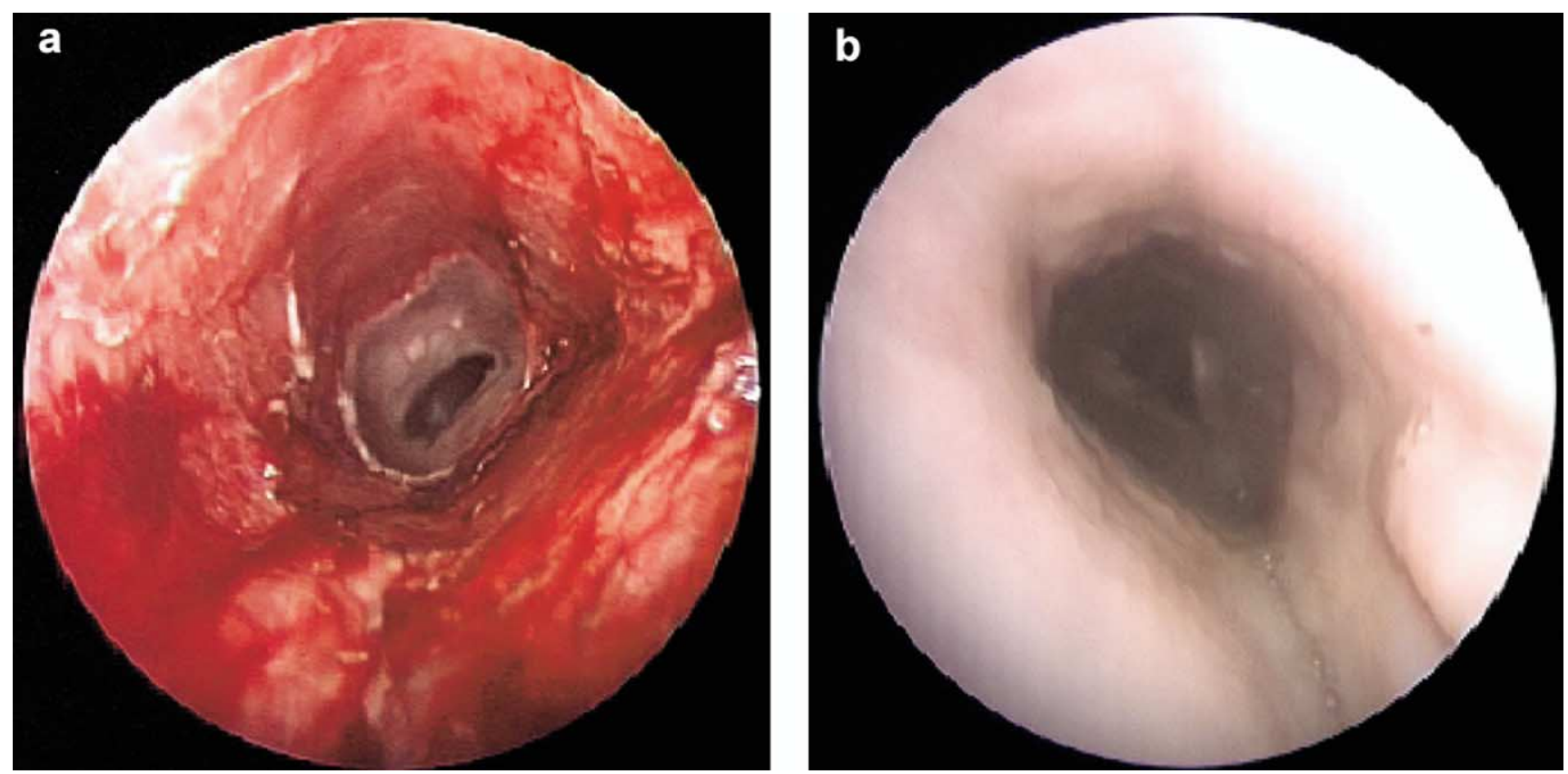

Figure E2. Endoscopic view. a, Endoscopic view after resection: mucosa was removed circumferentially over 5.5 cm in length. b, Endoscopic view showing complete re-epithelialization (4-month follow-up): cicatricial mucosa and absence of stenosis. This animal underwent three dilatation procedures. 
a

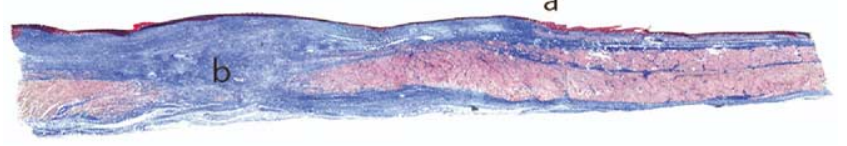

Figure E3. Healed esophagus. Longitudinal section of healed esophagus after 4.4-cm long EMR (Masson trichrome staining) showing distal section margin with interruption of lamina muscularis mucosae (a) and area of transmural fibrosis (b).

TABLE E1. Histologic analysis of the healed esophagi

\begin{tabular}{lccc}
\hline Group & Resection (cm) & Absent LMM (cm) & $\begin{array}{c}>50 \% \text { MP fibrosis } \\
\text { length (cm) }\end{array}$ \\
\hline 1 & 2.2 & $2.2(2.0-2.6)$ & $0.6(0.4-0.8)$ \\
2 & 3.3 & $2.9(2.6-3.3)$ & $0.9(0.9-1.0)$ \\
3 & 4.4 & $3.8(2.9-4.7)$ & $0.9(0.5-1.4)$ \\
4 & 5.5 & $4.1(3.0-5.9)$ & $1.3(0.7-2.1)$ \\
\hline
\end{tabular}

Values are means with ranges given in parentheses. $L M M$, Lamina muscularis mucosae; $M P$, muscularis propria. 